\title{
EEG-Based Demarcation of Yogic and Non-Yogic Sleep Patterns Using Power Spectral Analysis
}

\author{
Basavaraj Hiremath, Center for Medical Electronics and Computing, M.S Ramaiah Institute of Technology, Bangalore, \\ India \\ Natarajan Sriraam, Center for Medical Electronics and Computing, M.S Ramaiah Institute of Technology, Bangalore, India \\ (iD) https://orcid.org/0000-0003-3790-3900 \\ B. R. Purnima, Center for Medical Electronics and Computing, M.S Ramaiah Institute of Technology, Bangalore, India \\ Nithin N. S., Center for Medical Electronics and Computing, M.S Ramaiah Institute of Technology, Bangalore, India \\ Suresh Babu Venkatasamy, SVYASA University, India \\ Megha Narayanan, Center for Medical Electronics and Computing, M.S Ramaiah Institute of Technology, Bangalore, India
}

\begin{abstract}
Electroencephalogram (EEG) signals resulting from recordings of polysomnography play a significant role in determining the changes in physiology and behavior during sleep. This study aims at demarcating the sleep patterns of yogic and non-yogic subjects. Frequency domain features based on power spectral density methods were explored in this study. The EEG recordings were segmented into $1 \mathrm{~s}$ and $0.5 \mathrm{~s}$. EEG patterns with four windowing scheme overlaps $(0 \%, 50 \%, 60 \%$, and $75 \%)$ to ensure stationarity of the signal in order to investigate the effect of the pre-processing stage. In order to recognize the yoga and non-yoga group through N3 sleep stage, non-linear KNN classifier was introduced and performance was evaluated in terms of sensitivity and specificity. The experimental results show that modified covariance PSD estimate is the best method in classifying the sleep stage N3 of yogic and non-yogic subjects with 95\% confidence interval, sensitivity, specificity, and accuracy of $97.3 \%, 98 \%$, and $97 \%$, respectively.
\end{abstract}

\section{KEYWORDS}

Electroencephalogram (EEG), KNN, Power Spectral Density (PSD), Yoga

\section{INTRODUCTION}

Sleep is a spontaneously occurring phase of mind and body, marked by changed state of cognizance, diminished sensory activity, inhibition of almost all voluntary muscles and minimized external encounters. During sleep, the brain uses considerably less energy than when awake, notably during non-rapid eye movement (NREM) sleep. Humans secrete growth hormone surges in slow-wave sleep. Sleep is broadly classified into: rapid eye movement (REM) sleep and NREM. NREM Stage 1(Wake) is focused on whether your eyes are closed or open. Alpha and beta waves, primarily beta waves, are active when awake with eye-opened. When people get dizzy and close eyes, the prevailing pattern is 
the alpha wave Getting up from Stage N1 is easy One spends at least less than 10 minutes in Stage N1 sleep in the first sleep cycle. It makes up about $5 \%$ of total sleep duration. Stage 2 sleep usually takes approximately 25 minutes in the immediate cycle and extends by each subsequent cycle, gradually about $50 \%$. Stage 3 is the deepest sleep stage and is distinguished by relatively slow frequency with signals of higher magnitude termed as delta waves. It's the toughest level to wake up from. REM sleep is an active brain sleep cycle, but the body is immobilized. During REM sleep, intense dreams appear and the increase in the heart rate and breathing rate is observed (National Institute of Neurological Disorders and Stroke, 2019).

Yoga is a mind and body activity of ancient Indian tradition with a 5,000-year history. Specific yoga techniques incorporate physical asanas, methods of respiration, and meditation or relaxation. If people with insomnia practice yoga every day, they sleep longer, sleep quicker, and gets back to sleep quicker than normal if they got up in the middle of the night (The National Sleep Foundation, 2020). A national survey reported that more than 55 percent of yoga practitioners felt it assisted in getting better sleep (Alyson Ross, 2014). Research by Kalyan Maity et al. (2018) focused on examining the positive impacts of Sleep Special Technique (SST) on the quality of sleep, anxiety and well-being of fit young yoga practitioners. The experimental group received 1 month of (SST) training and there was no reference to SST from the control group. Substantial reductions in the global Pittsburgh sleep quality index (PQSI) value, perceived stress scale value and dramatic development in all Quality - of - life (QOL) scores were recorded after one month of SST practice. SST training of one month has a constructive influence on the entire quality of sleep, mental and physical health. Sarika Chaudhari et al. (2013) conducted a cross-sectional study to assess the influence of practicing yoga in the older people on quality of sleep and life quality. Overall PSQI score was less in yoga group than control group score. The Yoga group also had QOL scores than the control group. Practicing yoga for a long time by older people is aligned with little sleep disruptions and improved sleep quality and these findings are consistent with other studies involving only 180 days of yoga practice.

The research carried out by Li-Li Wang et al. (2016) was intended to assess EEG sleep efficiency from last night. Sleep trial was conducted to receive EEG signals from eight subjects under good, normal and poor sleep quality conditions. Taking into account the five different EEG frequency bands, the influence of sleep quality on the EEG expressed primarily in one or more frequency bands. The widely used EEG feature i.e., PSD was extracted in five frequency bands following the short-time Fourier transform. From the research observations, Gamma band is found to be the primary frequency band for determining sleep efficiency. Research by Rimpee Verma et al. (2018) helps people understand the sleep condition called a rapid eye behavior condition, the EEG signal's significance. The study allows the reader to have better information about sleep disorder, as well as its forms about EEG signals how it allows in sleep disorder diagnosis. The signal's PSD was determined by using Welch technique, whereupon the region contributing to delta, theta, alpha and beta bands were measured by using Trapezoidal Integration approach for evaluating average power. Power in the delta band has been reported to be greater for normal case however it is weakest in REM Sleep Behavior Disorders mostly during REM period.

A research by Massachusetts General Hospital (MGH) and Beth Israel Deaconess Medical Center (BIDMC) showed that a significant improvement in many areas resulted from the deep physiological condition that could be accomplished with the aid of yoga (The National Sleep Foundation, 2020). A regular yoga exercise relieves pain and anxiety. It also possesses the potential to diminish the amounts of hydrocortisone and relieve stress, all of which are exacerbated in anxiety or depression sufferers. Yoga triggers the nervous system which is parasympathetic. That's what makes you feel clam in your mind and relax the body, leading to improved sleep. Issues such as snoring and sleep apnea are caused by inappropriate respiration, especially hyperventilation. Snoring is almost always triggered by tension, problems with the sinus, overweight or issues with breathing, whereas sleep apnea is a classic indication in people with stressed, nervous or pulmonary abnormalities. Pranayama techniques will address all of these concerns, leading to enhanced sleep quality. 
Researchers have evaluated the influence of yoga on sleep with the cross-sectional studies. Analyzing the sleep behavior in the yoga and non-yoga group using the EEG is the novelty of this study. Having this context, the objective is to prove that the sleep pattern differs in yoga and nonyoga group and yoga practitioners get quicker and better sleep compared to non-yoga practitioners. This is to be achieved by using PSD based features as a robust approach for identifying the yogic and non-yogic deep sleep wave patterns.

To the best of author's knowledge, no attempts have been made to make use of EEG based demarcation of yogic and non-yogic sleep patterns. This proposed study suggests the usage of electroencephalogram(EEG) signals as a quantitative indicator to demarcate yogic and non-yogic group sleep patterns. Spectral density features based on Lomb-Scargle periodogram, Welch's spectral estimation, multitaper spectral estimation, covariance and modified covariance methods were investigated and the best PSD features identified were used to classify yogic and non-yogic group sleep patterns using KNN classifier. EEG being a non-stationarity signal, stationarity condition was preserved by applying $0.5 \mathrm{~s}$ and $1 \mathrm{~s}$ windowing with and without overlapping of EEG patterns for the analysis.

\section{METHOD AND MATERIALS}

\section{Data Description}

All-night PSG sleep recordings were provided by Swamy Vivekananda Yoga Anusandhana Samsthana, Bengaluru, India. It was a collection of 18 polysomnographic recordings collected at S-VYASA Institute's Sleep Laboratory, which included EEG channels, Electrooculogram (EOG) channels, photoplethysmogram (PPG) channel and other electrophysiological signals. Due ethical clearance was obtained and subject consent form was collected before the commencement of the study. In the present study, only EEG was considered. Amongst the 18 healthy subjects, nine of those were trained yoga practitioners and the rest were not. The overall length of all recordings combined for each subject was approximately $108 \mathrm{~h}$ with an average sleep duration of about $6 \mathrm{~h}$ '. The subjects were men and aged between 18 to 24 years, 21 years on average. A standard hypnogram describing the classification of sleep stages for each overnight sleep recording was obtained. Sampling or resampling of all signals was performed to $500 \mathrm{~Hz}$. A notch filter with a $50 \mathrm{~Hz}$ notch frequency, a $0.3 \mathrm{~Hz}$ high-pass filter and a $30 \mathrm{~Hz}$ low-pass filter were applied on EEG signals to eliminate noise and artifacts.

\section{MATERIALS}

\section{Power Spectral Density}

It is a well-established method of signal processing which is commonly used. Power spectral density is defined as the signal power distribution over frequency (Dressler et al., 2014; Rajak et al., 2016). It shows the strength of the energy as a frequency function (Sukhada A. Unde, 2014). Power spectral density analysis is often used for quantification of EEG signals (Dressler et al., 2014; Rajak et al., 2016). A sensible way to identify periodicity within the waveforms and determination of the relative energy content of the periodicities are given by this mathematical method of frequency understanding of various waveforms which is power spectral density analysis (Rajak et al., 2017).

\section{Lomb-Scargle Periodogram}

The Lomb-Scargle Periodogram is a widely used statistical tool designed to locate and test weak periodic signals in otherwise random, unevenly sampled observations . The standard periodogram formula was modified by Scargle to first find a time delay $\tau$ such that the pair of sinusoids would be mutually orthogonal at sample times $t_{j}$, instead of only taking dot products of data with sine and 
cosine waveforms, and even modified for the potentially unequal powers of the two basis functions, to achieve a better estimation of the power at a frequency.

\section{Welch's Spectral Estimation}

The Welch Periodogram is obtained by splitting the time signal into successive sections, forming the periodogram for each section, and averaging. This is to say, it's just an average of periodograms across time. This is an improvement on the standard periodogram spectrum estimating method and on Bartlett's method, in that it reduces noise in the estimated power spectra in return for reducing the frequency resolution. Because the process is wide-sense stationery and Welch's system employs PSD estimates of various segments of the time series, the updated periodograms represent approximately uncorrelated estimates of the true PSD and the variability is reduced by averaging.

Consider a sequence $\left\{x_{d}(n)\right\}, d=1,2,3, L$ are the signal intervals with a data window $w(n)$ and the length of each interval is M. Thus, according to Welch, the PSD is given by (Peter, 1967; Sabraj, 2016)

$$
\hat{P}_{d}(f)=\frac{1}{M U} \sum_{n=0}^{M-1} x_{d}(n) w(n) e^{-j 2 \pi f n}
$$

where $U$ is the normalization factor (Peter, 1967; Sabraj, 2016) for the power in the window function. The average over these modified periodograms gives the welch power spectrum (Peter, 1967; Sabraj, 2016) i.e.,

$$
\hat{P}_{\text {Welch }}(f)=\frac{1}{L} \sum_{i=0}^{L-1} \hat{P}_{d}(f)
$$

Where $\hat{P}_{\text {welch }}$ is the periodogram of the EEG signal of each interval.

\section{Multitaper Spectral Estimation}

The periodogram is not a reliable estimator of a wide-sense stationary process 's true power spectral density. To provide a clear PSD estimate, the multitaper approach combines modified periodograms obtained with the mutually orthogonal taper family. The Multi-Taper method of spectral analysis is used for the study of a time series, which is assumed to contain both continuous and singular components. This method aims at reducing the variance in spectral estimates by using a small set of tapers instead of the single data taper or spectral window used in Blackman-Tukey methods. A set of independent power spectrum estimates are calculated, by pre-multiplying the data by orthogonal tapers built to reduce the spectral leakage due to the finite length of the dataset.

\section{Covariance Method}

Welch 's approach is a specialized version with a single line, triangular or rectangular windowing with $0 \%$ overlap. The covariance approach fits the autoregressive (AR) linear prediction filter model to the signal that is presumed to be the outcome of the AR system guided by white noise by lessening the forward prediction error only in least square. The obtained spectral estimate is the squared measure of this AR model's frequency response. This approach is inexpensive on computation. The corresponding variation can be relatively high.

Let $\mathrm{y}(\mathrm{n})$ be a wide-sense stationary random process achieved with the system function $\mathrm{A}(\mathrm{z})$ by filtering white noise of variance e. If $P_{y}\left(e^{j \omega}\right)$ is the power spectral density of $y(n)$, then 


$$
P_{y}\left(e^{j w}\right)=\frac{e}{\left|A\left(e^{j w}\right)\right|^{2}}=\frac{e}{\left|1+\sum_{K-1}^{P} a(k) e^{-j w k}\right|^{2}}
$$

Since the approach involved an all-pole model to categorize the input signals, the right selection of model order $\mathrm{p}$ is essential.

\section{The Modified Covariance Method}

The Modified Covariance method is an advancement on both the Covariance method and the Burg method of funding the PSD. This autoregressive model assumes the collection of data can be used completely described by a linear combination of past outputs and driving noise. This method fits an AR model to the signal (Mark Lee, 2005). The modified covariance method anticipates the P coefficients, where $\mathrm{P}$ is the model order, by minimizing the forward and backward prediction errors in the least squares sense (Mark Lee, 2005):

$$
\begin{aligned}
& \hat{p}=0.5\left(\hat{p}^{f}+\wedge^{b}\right) \\
& \hat{p}^{f}=\frac{1}{N-P} \sum_{n=0}^{N-1-P}\left|y_{n}+\sum_{k=1}^{P} a_{k} y_{n-k}\right|^{2}
\end{aligned}
$$

and similarly, for $\hat{\rho}^{b}$, where $\mathrm{N}$ is the data length and $\mathrm{a}_{\mathrm{k}}$ is the $\mathrm{k}^{\text {th }} \mathrm{AR}$ coefficient.

It also does not suffer from the splitting of spectral lines, and provides even less distortion when compared with Covariance method. This is more demanding computationally than the Covariance method.

\section{Power Spectrum}

The power spectrum is generally known as the Fourier transform of the autocorrelation function. It makes use of linear interpolation and assumes a sample time same as the median of the differences among neighboring time points. Power spectrum detects a balance between the achievable spectral resolution for the entire signal length and the performance limitations arising from result from calculating large FFTs.

\section{Feature Extraction}

Features were obtained for a segmentation length of both $0.5 \mathrm{~s}$ and $1 \mathrm{~s}$ with various overlapping conditions, i.e. 0 per cent, 50 per cent, 65 per cent and 75 per cent overlap and the appropriate one chosen based on the classifier performance. Table 1 includes a list of features that are used in proposed work.

\section{Classifier}

The classifier predicts the respective class of independent features as the input to which a independent variable belongs (Pereira, 2009; Amin, 2017). A training dataset and a test data set are necessary for the classification. The trained classifier must construct the relation among classes as well as the respective features. The model employed for the classification in this study was the K-nearest neighbor $(\mathrm{KNN})$ classifier. It is a classifier which is non-linear. It is simple yet robust. It can deliver high-performance outcomes including for challenging applications (Mustafa, 2012). In order to decide the data belonging to which category, KNN uses a distance of features within a data set, a test sample 
Table 1. List of EEG features

\begin{tabular}{|c|l|}
\hline EEG Feature & \multicolumn{1}{c|}{ Description } \\
\hline Mean Frequency & $\begin{array}{l}\text { It is the ratio of sum of product of power spectrum of EEG } \\
\text { and frequency to the total power spectrum. }\end{array}$ \\
\hline Median Frequency & $\begin{array}{l}\text { The frequency which separates the power spectrum of EEG } \\
\text { into two parts with half the total power or equal amplitude. }\end{array}$ \\
\hline Maximum-to-minimum difference & $\begin{array}{l}\text { The difference between the maximum and minimum values } \\
\text { in EEG signal }\end{array}$ \\
\hline Peak-magnitude-to-RMS ratio & $\begin{array}{l}\text { The largest absolute value in x divided by the root-mean- } \\
\text { square (RMS) value of EEG signal. }\end{array}$ \\
\hline Root-mean-square level & The root-mean-square (RMS) level of the EEG signal \\
\hline Root-sum-of-squares level & The root-sum-of-squares (RSS) level of the EEG signal \\
\hline Spurious free dynamic range & $\begin{array}{l}\text { The strength of the fundamental signal divided by the } \\
\text { strength strongest spurious output signal. }\end{array}$ \\
\hline
\end{tabular}

class is classified by $\mathrm{k}$ nearest neighbor as per the majority class of k-nearest training samples (Amin, 2017). A group shall be created when the gap between the data is near. Many classes are established whenever the distance among the data points is farther (Mustafa, 2012).

\section{METHODOLOGY}

We obtained 18 subjects' pre-processed EEG sleep data with a comprehensive list of annotations for each. There were six EEG channels (F3A1, F4A2, C3A1, C4A2, O1A1, and O2A2) in the EEG recordings. In addition, each EEG channel had five sleep stages-W, N1, N2, N3, R. Sample estimation was conducted for the entire length of each subject's sleep study. Many instances of the same sleep stage have been recorded as the subject undergoes various sleep cycles during the overall sleep time. This work uses only the Sleep Stage N3 data, since most of the deep sleep occurs in this stage. The block diagram of the proposed methodology is shown in Figure 1. Every N3 sleep stage data samples are segmented with $1 \mathrm{~s}$ and $0.5 \mathrm{~s}$ window with various overlapping conditions. Six different PSD estimations were carried out on each segment and the features like mean frequency, median

Figure 1. Proposed schematic diagram for yoga- non-yoga group recognition

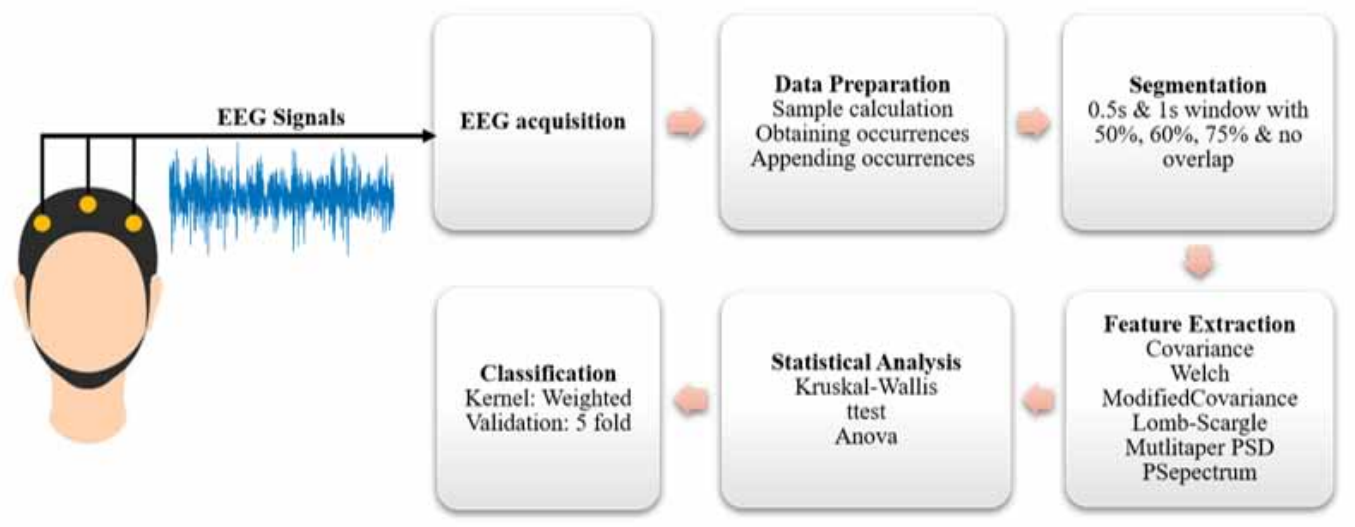


Figure 2. EEG-Raw data (Stage N3) of a yogic subject

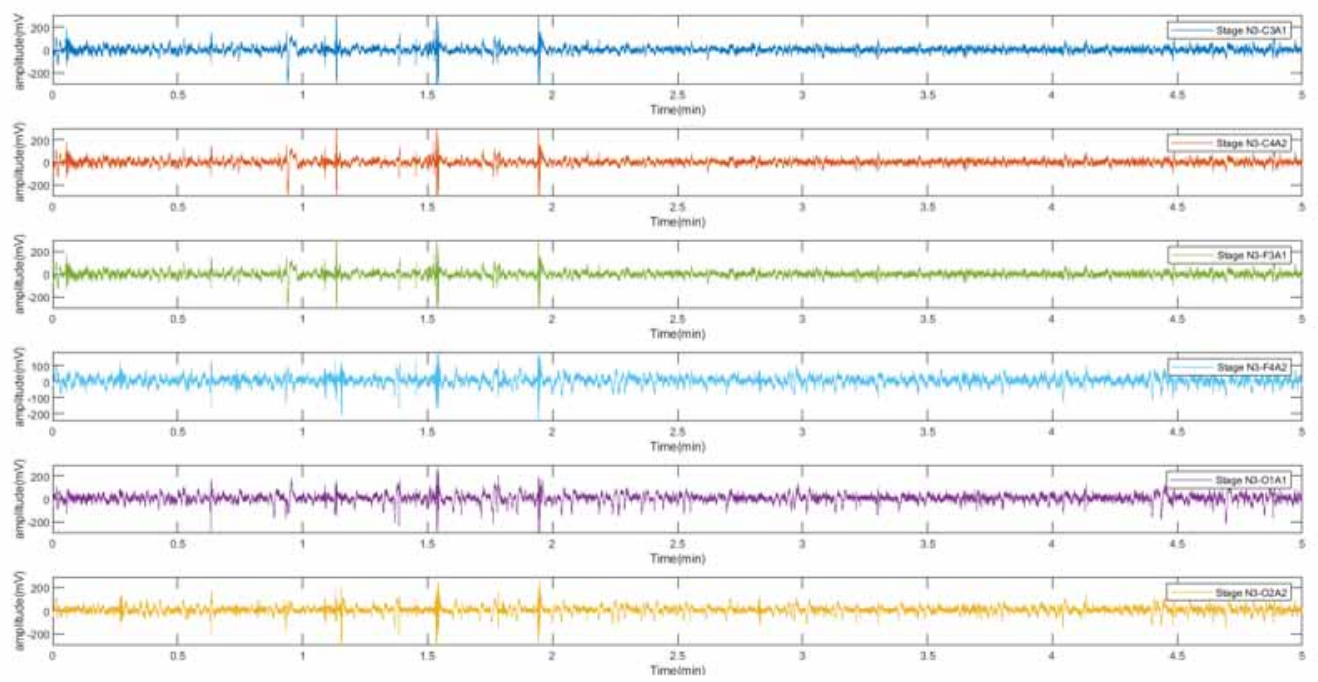

frequency, maximum-to-minimum difference, peak-magnitude-to-rms ratio, root-mean-square level, root-sum-of-squares level and spurious free dynamic range were extracted.

The statistical testing of the hypothesis was carried out using the p-value approach to ensure that the null hypothesis was rejected. The p-values obtained for different combinations of features are $<$ 0.05 , suggesting the presence of variations in the patterns of sleep. The box-plots of the extracted attributes showed fairly significant contradictions in the second quartile for stage N3 of yogic and non-yogic subjects, implying a clear contrast in the sleep patterns. The k-nearest neighbor algorithm has been used to classify the sleep pattern of stage N3 among yoga and non-yoga practitioners. The weighted classifier type which performs medium distinctions between classes, using a distance weight and 10 nearest neighbors. Sets of training and testing are planned in a ratio of 70 to 30 with a 5-fold cross validation. $70 \%$ data samples are randomly selected without substitution from each yogic set and non-yogic set for the training set and $30 \%$ from the test set.

\section{RESULTS \& DISCUSSION}

For evaluation purposes, EEG signals from all the channels of eighteen subjects were used to distinguish between the sleep patterns in this study. Figures 2 and 3 displays a contrast between EEG of a yoga and non-yoga practitioner with the sleep stage N3 extracted.

From the raw EEG plots of Sleep Stage N3 of yoga and non-yoga practitioner, it can be inferred that the yogic sleep pattern has less disturbances compared to the non-yogic sleep pattern. The EEG wave of stage N3 is comparatively slower in yogic than in non-yoga practitioner.

In this study EEG signals were segmented between two successive time windows into a number of time windows with different overlapping conditions. As illustrated in Figure 4, EEG data is loaded every 1 second. After which, the EEG data is segmented by a 1-s window with 0, 50, 60 per cent and 75 per cent overlap between two consecutive windows. The same sliding windows structure is followed for $0.5 \mathrm{~s}$ window as well.

EEGdata are loaded every 1 second. Then, a 2-s window with a 50\% overlap between two consecutive windows is used to segment the oltered and artifact-free EEGdata. 105 EEGfeatures are computed in each 2-s window to predict emotional states. We make a decision upon the three consecutive 2-s windows according to the majority rule. In other words, we make the orst decision 
Figure 3. EEG-Raw data (Stage N3) of a non-yogic subject

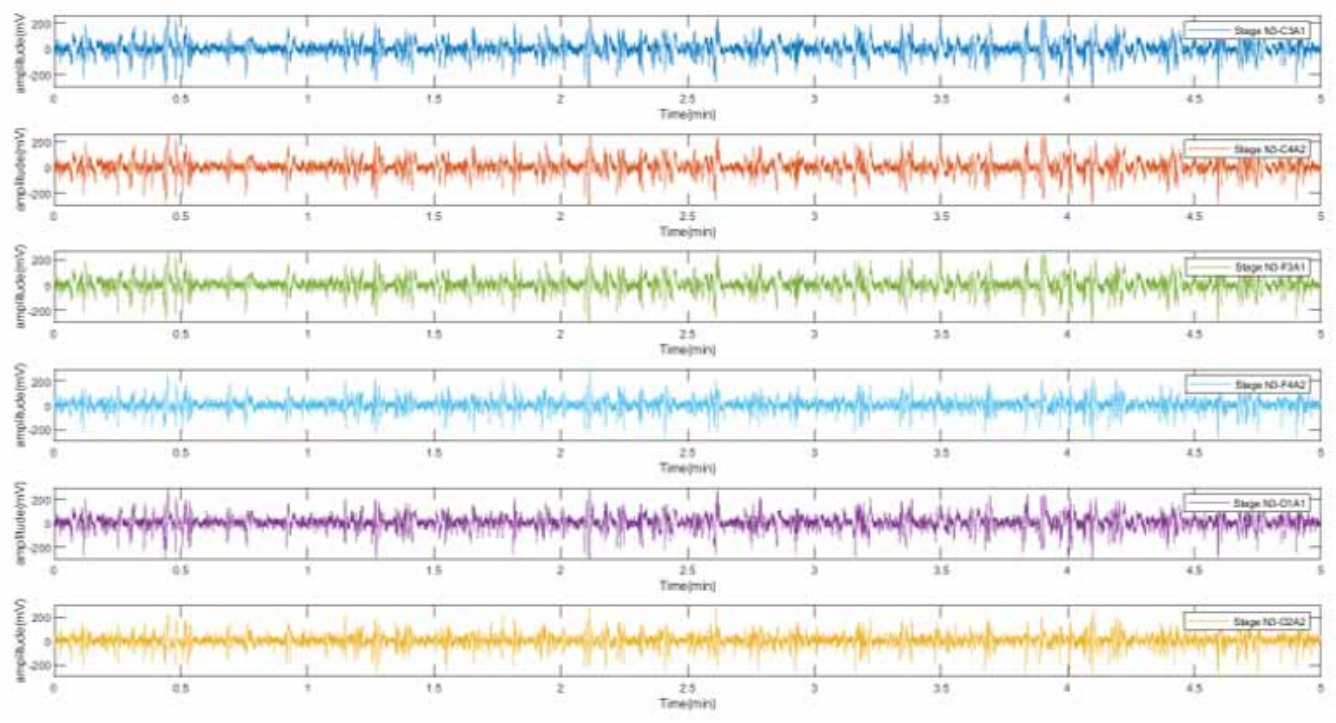

after EEGdata are continuously imported for 4 seconds. After that, emotional states are predicted every 1 second.

EEGdata are loaded every 1 second. Then, a 2 -s window with a50\% overlap between two consecutive windows is used to segment the oltered and artifact-free EEGdata. 105 EEGfeatures are computed in each 2-s window to predict emotional states. We make a decision upon the three consecutive 2-s windows according to the majority rule. In other words, we make the orst decision after EEGdata are continuously imported for 4 seconds. After that, emotional states are predicted every 1 second.

EEGdata are loaded every 1 second. Then, a 2-s window with a 50\% overlap between two consecutive windows is used to segment the ðltered and artifact-free EEGdata. 105 EEGfeatures are computed in each 2-s window to predict emotional states. We make a decision upon the three consecutive 2-s windows according to the majority rule. In other words, we make the ðrst decision after EEGdata are continuously imported for 4 seconds. After that, emotional states are predicted every 1 second.

EEGdata are loaded every 1 second. Then, a 2-s window with a 50\% overlap between two consecutive windows is used to segment the ðltered and artifact-free EEGdata. 105 EEGfeatures are computed in each 2-s window to predict emotional states. We make a decision upon the three consecutive 2-s windows according to the majority rule. In other words, we make the drst decision after EEGdata are continuously imported for 4 seconds. After that, emotional states are predicted every 1 second

In order to show channel wise demarcation between yogic and non-yogic group, feature plots with $1 \mathrm{~s}$ and $0.5 \mathrm{~s}$ windowing was shown. It can be inferred from the feature plots that the PSD features shows distinguishable difference between sleep patterns of yogic and non- yogic group. It can be further observed that the features values of both the groups differ greatly for all the channels. According to Figures 5 and 6, the median frequency values of the modified covariance PSD estimate are situated well above the origin, i.e., in the higher range, while the values for non-yoga lies close to the origin i.e., in the lower range. The trend repeats for all the channels. The pattern is similar for all other features as well. Hence, it can be concluded that the features for both the groups are distinct and strongly aids in classification. Table 2 and Table 3 shows the statistical analysis results 


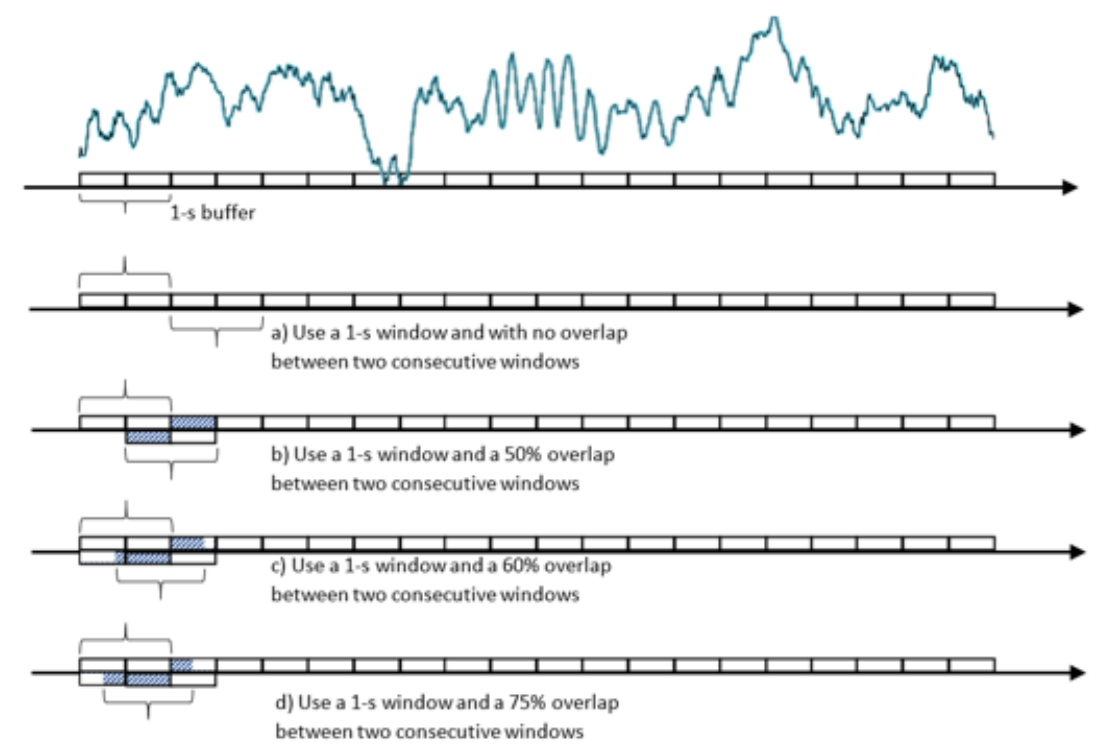

The p-tests were performed using Kruskal-Wallis test, ttest and Anova tests. The null hypothesis is that there is no relationship between the sleep stage N3 of Yoga and Non-Yoga practitioners, while the alternate hypothesis states the opposite. The $h$ value 1 and $p<0.05$ supports the strong rejection of null hypothesis. All the three p-tests indicates that there is a statistically very notable difference in the N3 stage of sleep with the $\mathrm{p}<0.05$ and $\mathrm{h}$ value of 1 for all the features extracted. The results suggest there exists a very notable difference between the Stage N3 sleep pattern of both the groups.

The box-plot is used as a descriptive statistic to summarize the differences in the sleep stages. The second quartile gives the median values of the selected features for the sleep stage N3. The box

Figure 5. Feature plots of the best PSD method for median frequency using $1 \mathrm{~s}$ window

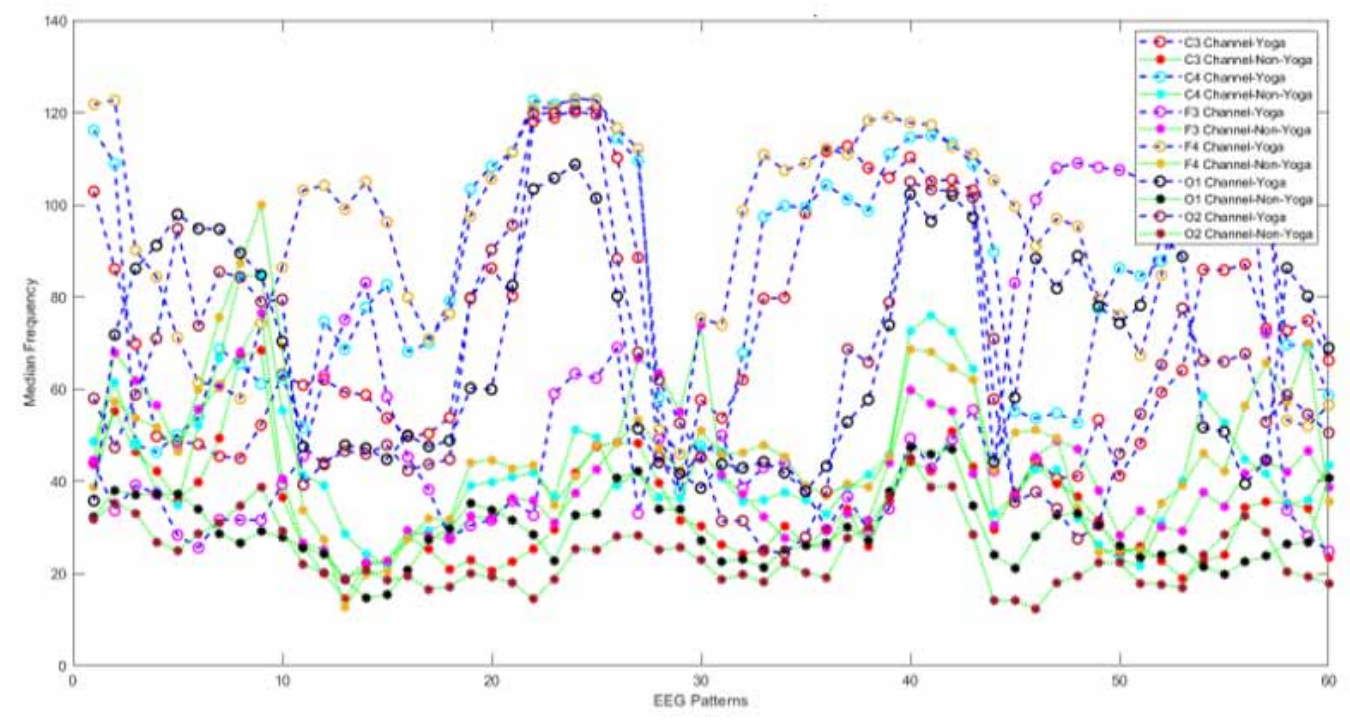


Figure 6. Feature plots of the best PSD method for median frequency using $0.5 \mathrm{~s}$ window

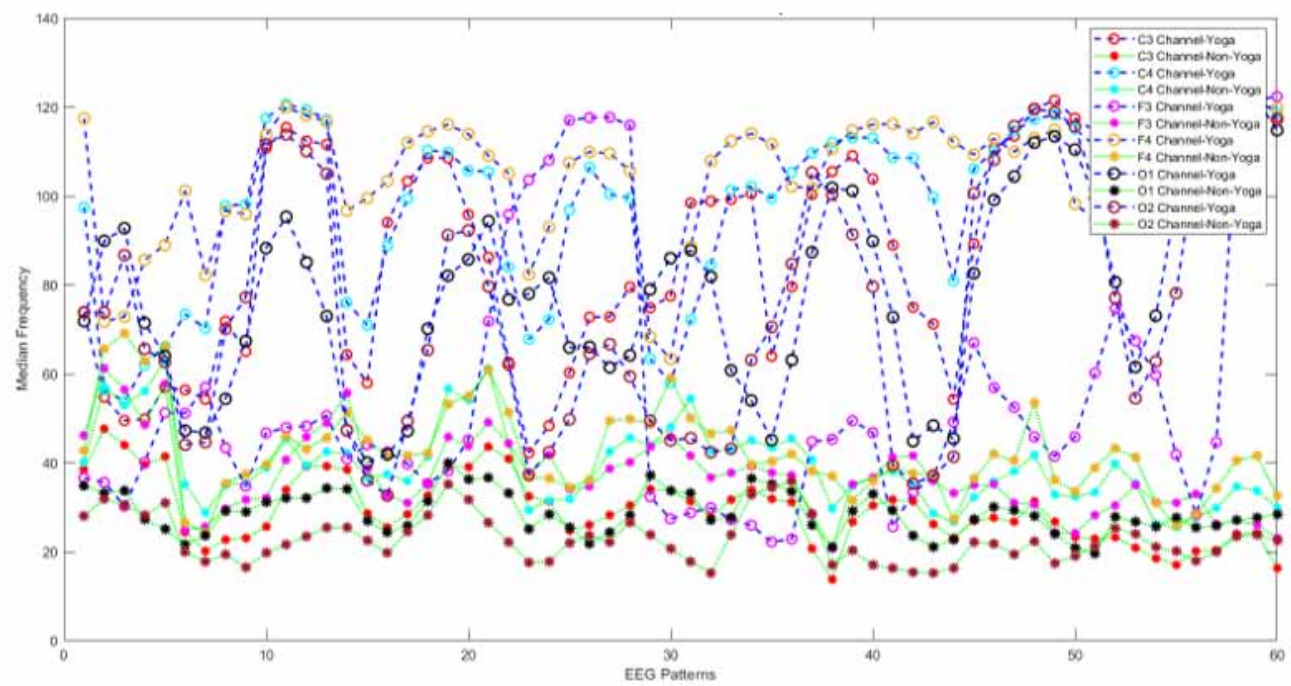

Table 2. Statistical analysis of the best PSD method using three different tests for $0.5 \mathrm{~s}$ window with (a) $0 \%$ (b) $50 \%$ (c) $60 \%$ (d) $75 \%$ overlap condition

\begin{tabular}{|c|c|c|c|c|c|c|c|c|c|}
\hline $\begin{array}{c}\text { Overlap } \\
\text { Condition }\end{array}$ & & & meanfreq & medfreq & max2mindiff & peak2rms & RMS & SFDR & RMS \\
\hline \multirow[t]{3}{*}{ 0\% (No) } & $\begin{array}{c}\text { Kruskal } \\
\text { Wallis }\end{array}$ & p-value & 0 & 0 & 0 & 3.33E-11 & 0 & $\begin{array}{l}7.92 \mathrm{E}- \\
12\end{array}$ & 0 \\
\hline & ttest & p-value & 0 & 0 & $1.60 \mathrm{E}-13$ & $6.39 \mathrm{E}-12$ & $\begin{array}{c}4.52 \mathrm{E}- \\
13\end{array}$ & $\begin{array}{c}1.20 \mathrm{E}- \\
27\end{array}$ & $\begin{array}{c}4.52 \mathrm{E}- \\
13\end{array}$ \\
\hline & Anova & p-value & 0 & 0 & $1.60 \mathrm{E}-13$ & $6.39 \mathrm{E}-12$ & $\begin{array}{c}4.52 \mathrm{E}- \\
13\end{array}$ & $\begin{array}{c}1.20 \mathrm{E}- \\
27\end{array}$ & $\begin{array}{c}4.52 \mathrm{E}- \\
13\end{array}$ \\
\hline \multirow[t]{3}{*}{$\mathbf{5 0} \%$} & $\begin{array}{c}\text { Kruskal } \\
\text { Wallis }\end{array}$ & $\mathrm{p}$-value & 0 & 0 & 0 & $1.27 \mathrm{E}-25$ & 0 & $\begin{array}{c}4.29 \mathrm{E}- \\
22\end{array}$ & 0 \\
\hline & ttest & p-value & 0 & 0 & $1.19 \mathrm{E}-264$ & $9.09 \mathrm{E}-25$ & $\begin{array}{l}1.43 \mathrm{E}- \\
25\end{array}$ & $\begin{array}{c}2.18 \mathrm{E}- \\
40\end{array}$ & $\begin{array}{l}1.43 \mathrm{E}- \\
25\end{array}$ \\
\hline & Anova & $\mathrm{p}$-value & 0 & 0 & $1.19 \mathrm{E}-264$ & $9.09 \mathrm{E}-25$ & $\begin{array}{c}1.43 \mathrm{E}- \\
25\end{array}$ & $\begin{array}{c}2.18 \mathrm{E}- \\
40\end{array}$ & $\begin{array}{c}1.43 \mathrm{E}- \\
25\end{array}$ \\
\hline \multirow[t]{3}{*}{$60 \%$} & $\begin{array}{c}\text { Kruskal } \\
\text { Wallis }\end{array}$ & p-value & 0 & 0 & 0 & $1.719 \mathrm{e}-32$ & 0 & $\begin{array}{l}3.95 \mathrm{E}- \\
280\end{array}$ & 0 \\
\hline & ttest & p-value & 0 & 0 & 0 & 0 & $1.30 \mathrm{e}-32$ & $\begin{array}{c}9.58 \mathrm{E}- \\
51\end{array}$ & $1.30 \mathrm{e}-32$ \\
\hline & Anova & p-value & 0 & 0 & 0 & 0 & $1.29 \mathrm{e}-32$ & $\begin{array}{c}9.58 \mathrm{E}- \\
51\end{array}$ & $1.29 \mathrm{e}-32$ \\
\hline \multirow[t]{3}{*}{$75 \%$} & $\begin{array}{c}\text { Kruskal } \\
\text { Wallis }\end{array}$ & p-value & 0 & 0 & 0 & 0 & 0 & 0 & 0 \\
\hline & ttest & $\mathrm{p}$-value & 0 & 0 & 0 & 0 & 0 & $\begin{array}{c}6.29 \mathrm{E}- \\
81\end{array}$ & 0 \\
\hline & Anova & p-value & 0 & 0 & 0 & 0 & 0 & $\begin{array}{c}6.29 \mathrm{E}- \\
81\end{array}$ & 0 \\
\hline
\end{tabular}


Table 3. Statistical analysis using three different tests for 1 s window with (a) $0 \%$ (b) $50 \%$ (c) $60 \%$ (d) $75 \%$ overlap condition

\begin{tabular}{|c|c|c|c|c|c|c|c|c|c|}
\hline $\begin{array}{l}\text { Overlap } \\
\text { Condition }\end{array}$ & & & meanfreq & medfreq & max2mindiff & peak2rms & RMS & SFDR & RMS \\
\hline \multirow[t]{3}{*}{ 0\% (No) } & $\begin{array}{c}\text { Kruskal } \\
\text { Wallis }\end{array}$ & $\mathrm{p}$-value & 0 & 0 & 0 & $7.22 \mathrm{E}-34$ & 0 & $\begin{array}{c}2.46 \mathrm{E}- \\
266\end{array}$ & 0 \\
\hline & ttest & p-value & 0 & 0 & $7.29 \mathrm{E}-216$ & 4.67E-70 & $\begin{array}{c}2.89 \mathrm{E}- \\
215\end{array}$ & $\begin{array}{l}1.67 \mathrm{E}- \\
217\end{array}$ & $\begin{array}{c}2.89 \mathrm{E}- \\
215\end{array}$ \\
\hline & Anova & p-value & 0 & 0 & $7.29 \mathrm{E}-216$ & 4.67E-70 & $\begin{array}{c}2.89 \mathrm{E}- \\
215\end{array}$ & $\begin{array}{c}1.67 \mathrm{E}- \\
217\end{array}$ & $\begin{array}{c}2.89 \mathrm{E}- \\
215\end{array}$ \\
\hline \multirow[t]{3}{*}{$\mathbf{5 0} \%$} & $\begin{array}{c}\text { Kruskal } \\
\text { Wallis }\end{array}$ & p-value & 0 & 0 & 0 & $2.43 \mathrm{E}-74$ & 0 & 0 & 0 \\
\hline & ttest & p-value & 0 & 0 & 0 & $8.60 \mathrm{E}-149$ & 0 & 0 & 0 \\
\hline & Anova & p-value & 0 & 0 & 0 & $8.60 \mathrm{E}-149$ & 0 & 0 & 0 \\
\hline \multirow[t]{3}{*}{$60 \%$} & $\begin{array}{c}\text { Kruskal } \\
\text { Wallis }\end{array}$ & $\mathrm{p}$-value & 0 & 0 & 0 & $1.46 \mathrm{E}-102$ & 0 & 0 & 0 \\
\hline & ttest & $\mathrm{p}$-value & 0 & 0 & 0 & $1.77 \mathrm{E}-193$ & 0 & 0 & 0 \\
\hline & Anova & p-value & 0 & 0 & 0 & $1.77 \mathrm{E}-193$ & 0 & 0 & 0 \\
\hline \multirow[t]{3}{*}{$75 \%$} & $\begin{array}{c}\text { Kruskal } \\
\text { Wallis }\end{array}$ & $\mathrm{p}$-value & 0 & 0 & 0 & $6.22 \mathrm{E}-160$ & 0 & 0 & 0 \\
\hline & ttest & $\mathrm{p}$-value & 0 & 0 & 0 & $1.27 \mathrm{E}-305$ & 0 & 0 & 0 \\
\hline & Anova & $\mathrm{p}$-value & 0 & 0 & 0 & $1.27 \mathrm{E}-305$ & 0 & 0 & 0 \\
\hline
\end{tabular}

plots in the Figures 7 and 8 clearly show that the median value varies significantly for all the features which indicates that the data sample is skewed and there exists a remarkable distinction between the N3 sleep stage of yoga and non-yoga subjects.

Figures 9 and 10 compares accuracy of the KNN classifier which was modeled using the features extracted from $0.5 \mathrm{~s}$ and $1 \mathrm{~s}$ window length for four different overlap conditions. Although we observed a slight increase in the performance for all the PSD methods, there was not much of a difference for welch and multitaper methods. When the ensemble of both $0.5 \mathrm{~s}$ and $1 \mathrm{~s}$ window was done as shown in Figure 11, we observed that the classifier performance didn't improve but was similar to 0.5 window performance. Since, $1 \mathrm{~s}$ window performed slightly better than $0.5 \mathrm{~s}$ window, an ensemble of all the overlapping conditions of $1 \mathrm{~s}$ was performed and surprisingly the accuracy obtained was higher than the best overlapping method (75\%) as depicted in Figure 12.

The performance of the KNN classifier was high when trained for features of Modified covariance PSD estimate with an accuracy of around $97.3 \%$ with an F1-score of 0.98 which is a very good score in detecting False Negatives and False Positives. Also, the model has an Area under ROC curve (AUC) of 0.99 which is an excellent score in determining how much model is capable of distinguishing between classes. The ROC curve quite closely follows the left-hand border and then the top border of the ROC space indicates the tests conducted are precise. These results suggest that the Stage N3 of both the groups differ in great amount and can be significantly classified. 
Figure 7. Box plot indicating median of feature values of best PSD estimate for yoga (red) and nonyoga (blue) with 1s window for the best overlapping condition $(75 \%)$

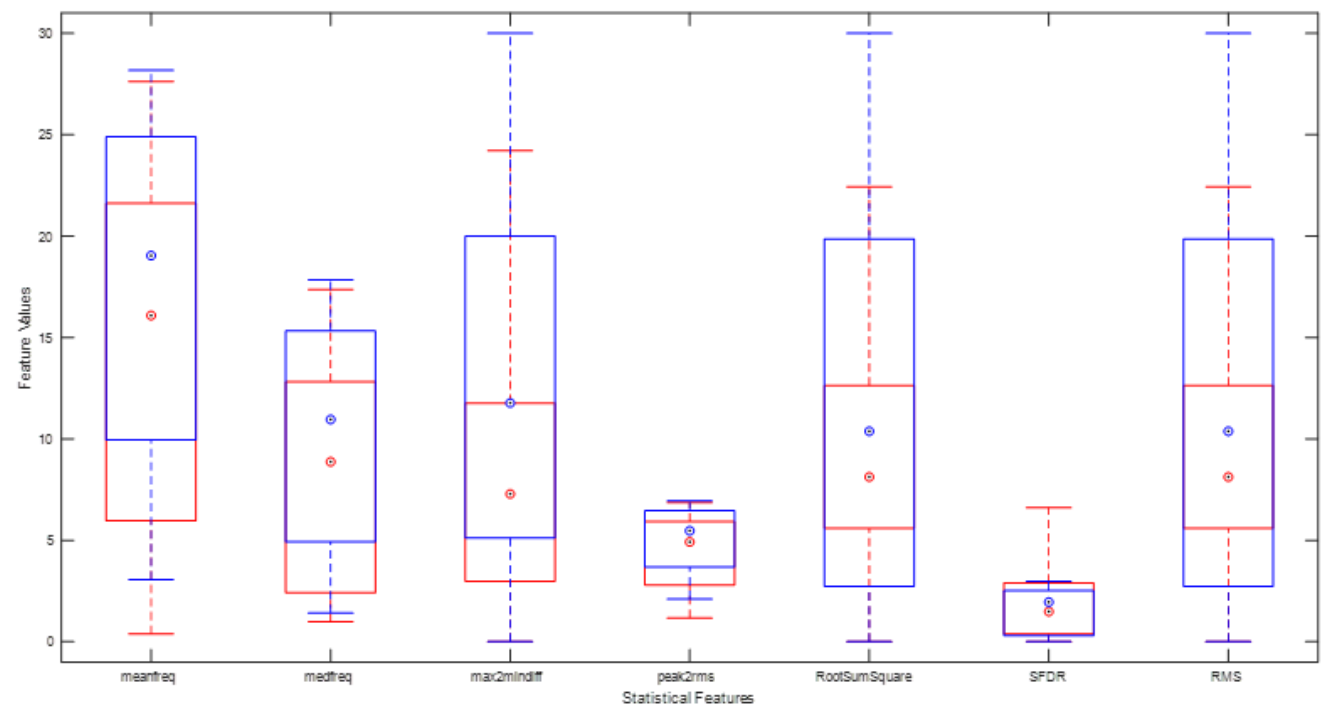

Figure 8. Box plot indicating median of feature values of best PSD estimate for yoga (red) and nonyoga (blue) with $0.5 \mathrm{~s}$ window for the best overlapping condition $(75 \%)$

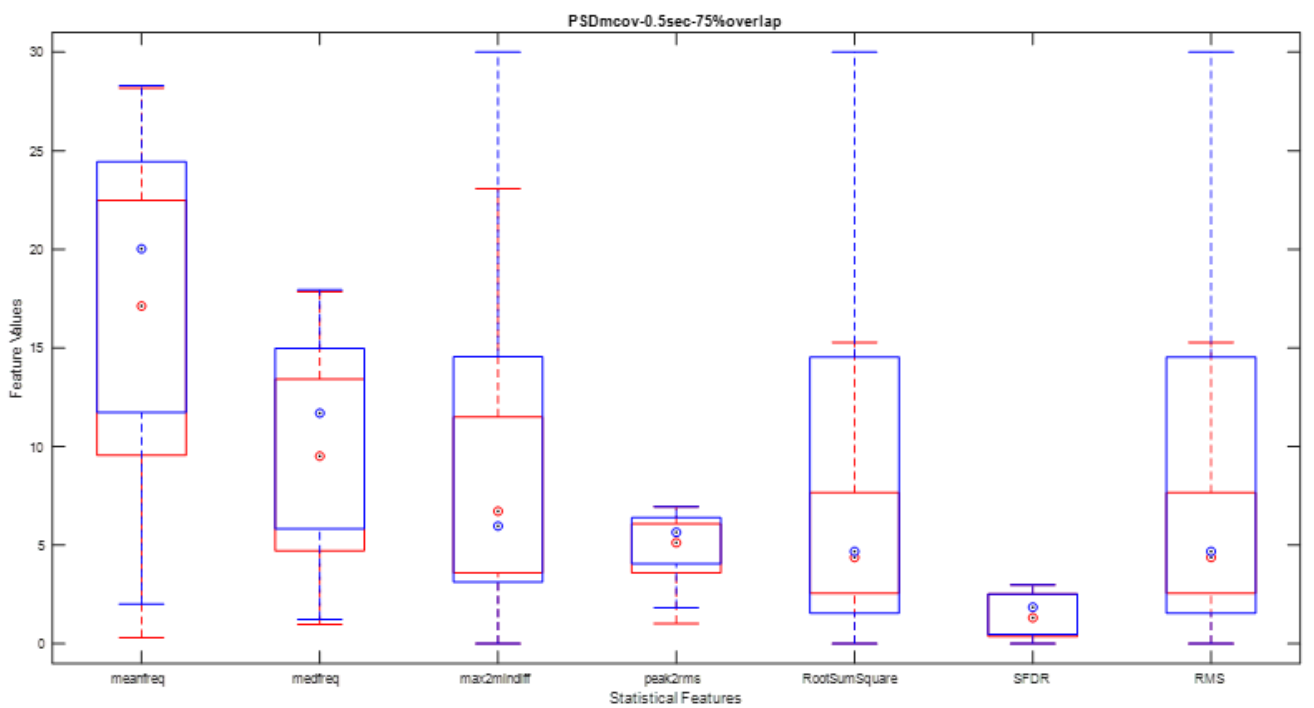


Figure 9. Classifier performance for $0.5 \mathrm{~s}$ window

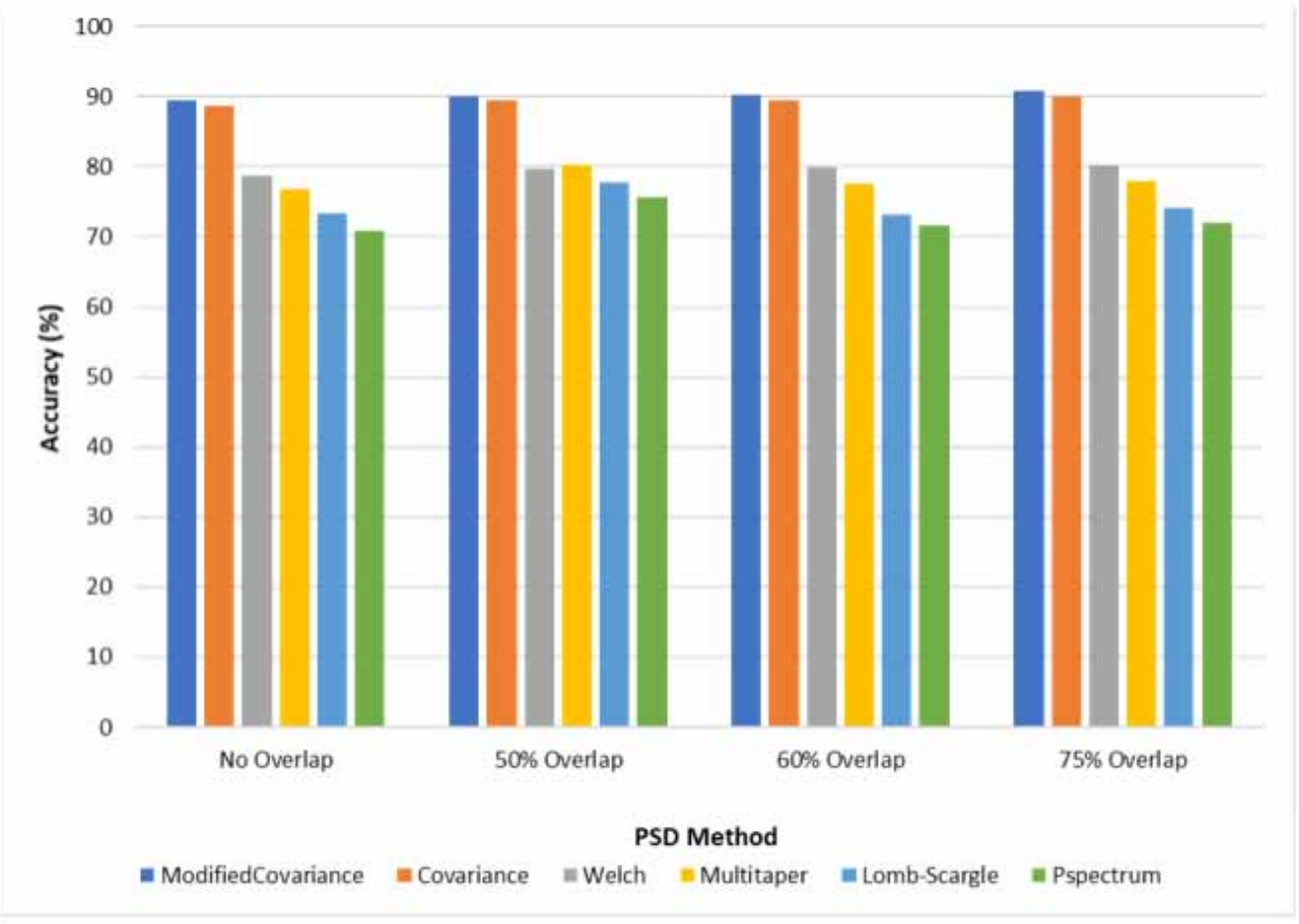


International Journal of E-Health and Medical Communications

Volume $12 \cdot$ Issue 6 • November-December 2021

Figure 10. Classifier performance for 1s window

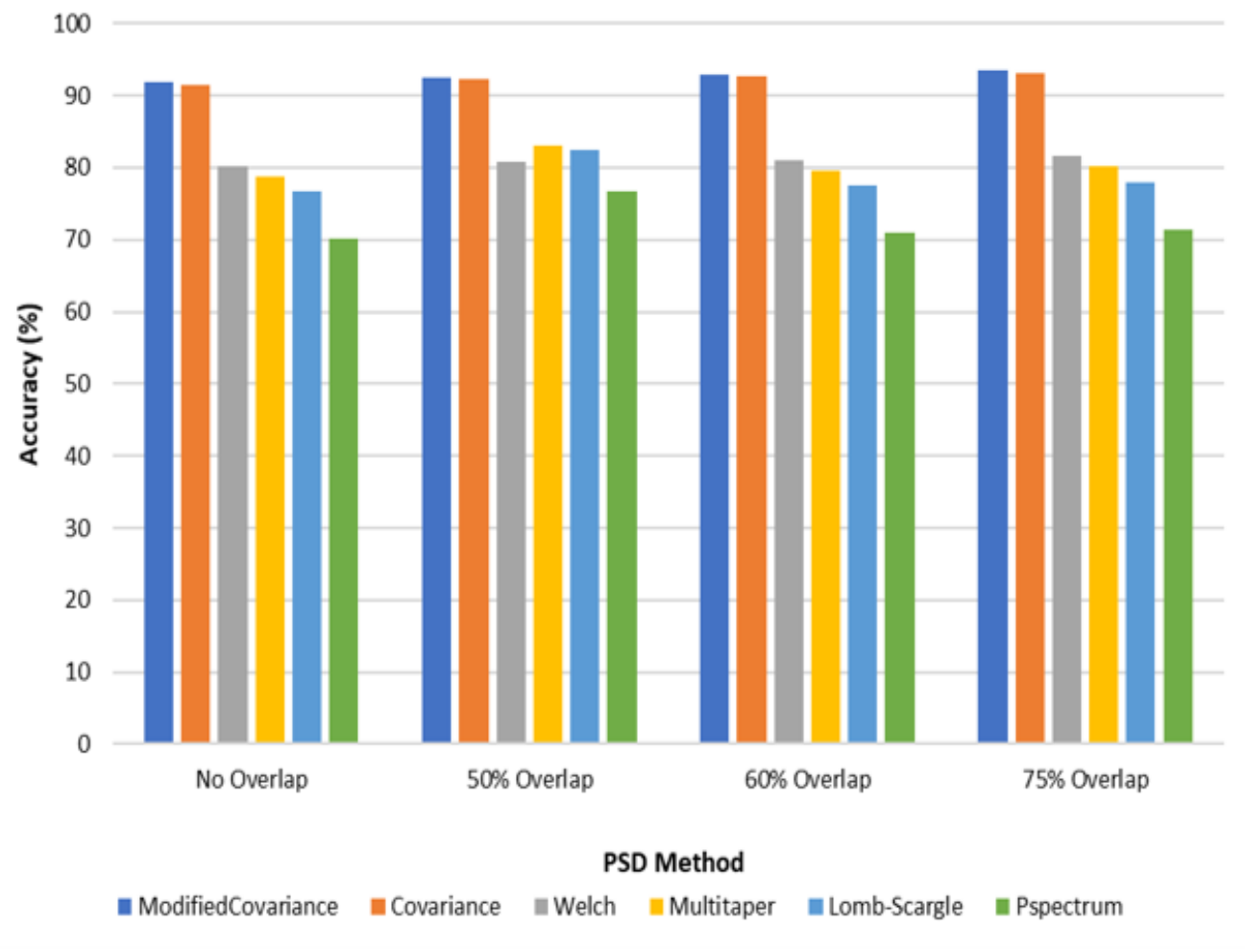


Figure 11. Classifier performance for ensemble of $0.5 \mathrm{~s}$ and $1 \mathrm{~s}$ window

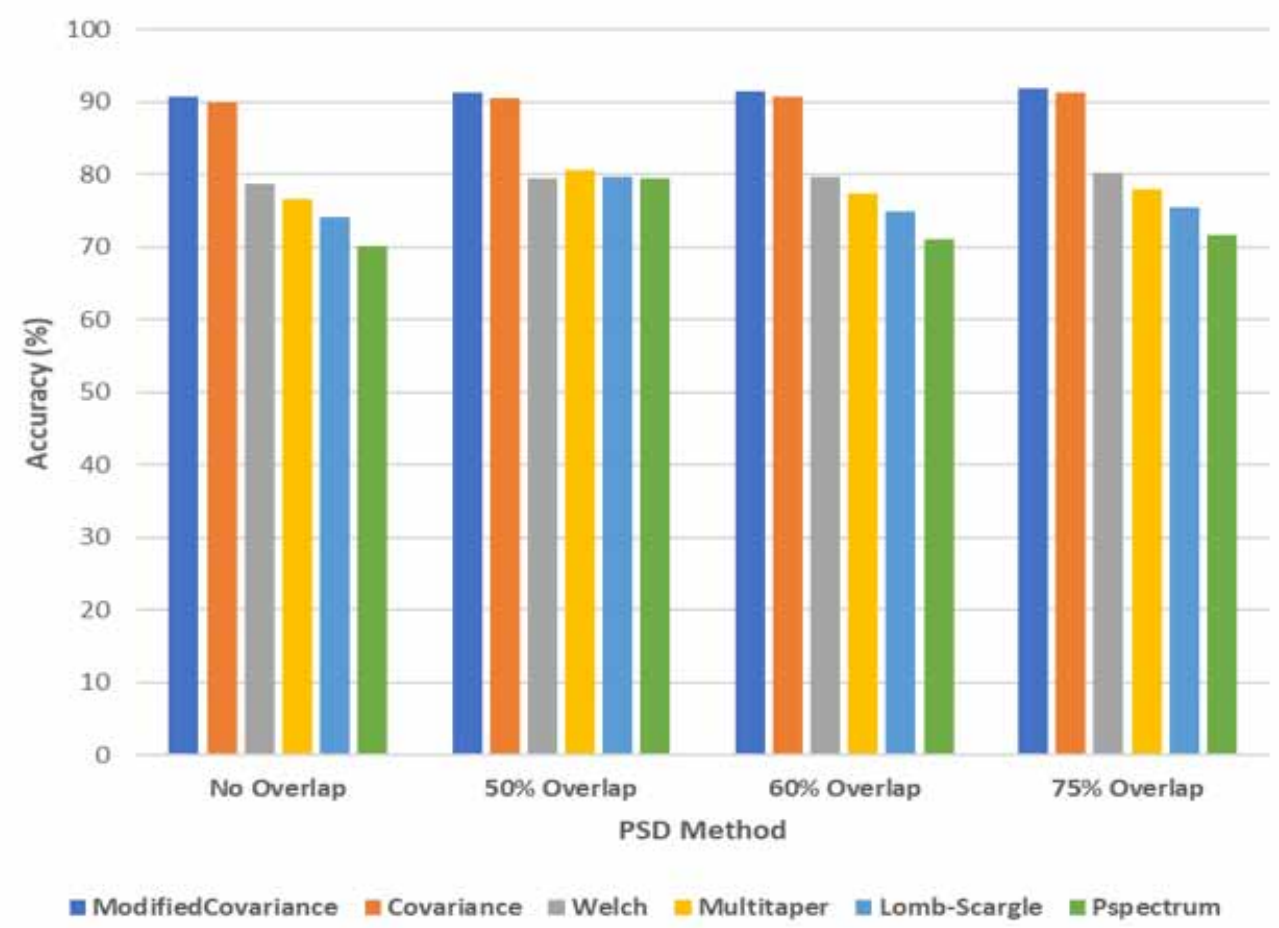




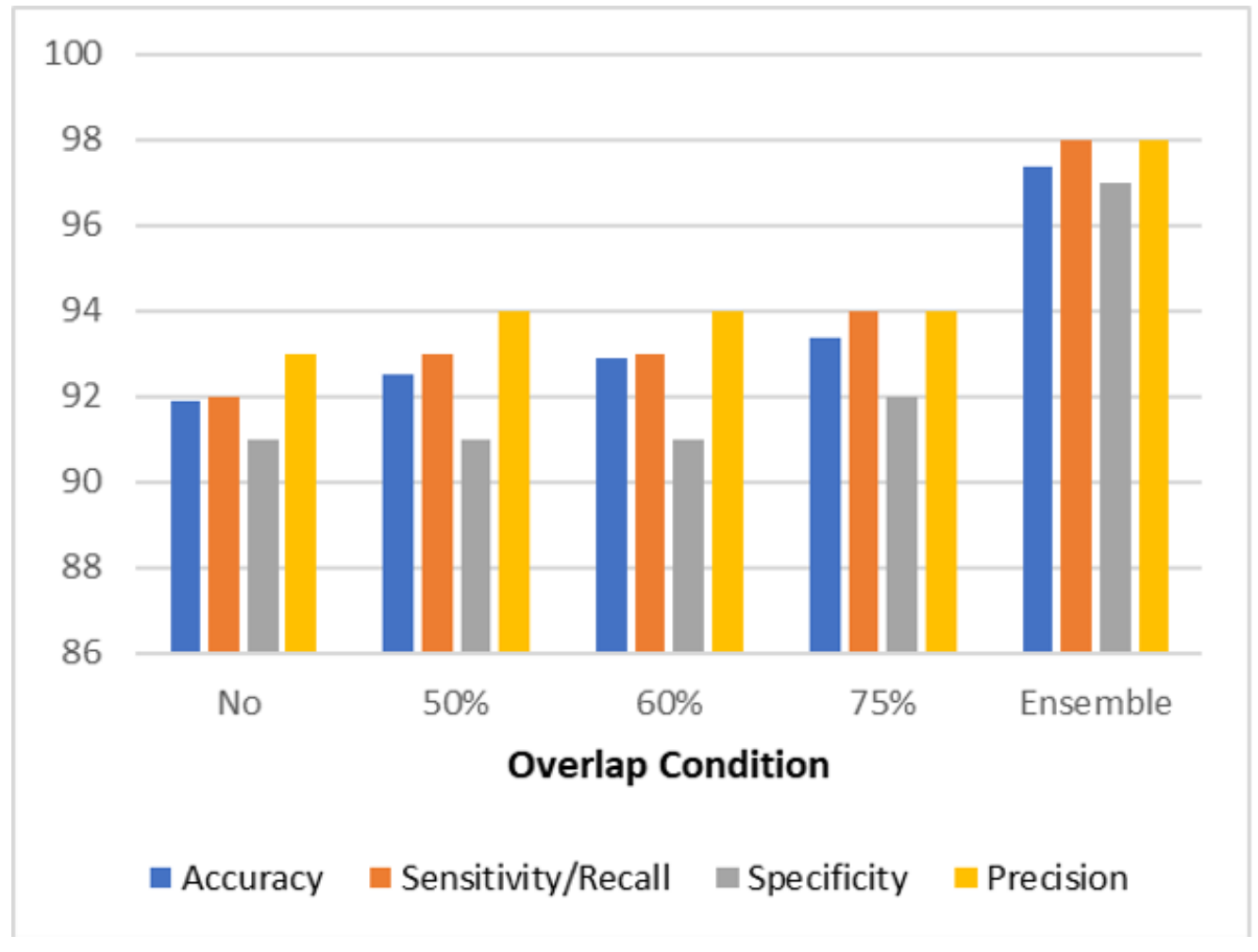

\section{CONCLUSION}

This work aims at understanding the impact of yoga on the patterns of sleep and how it helps to improve sleep. In this analysis, among the studied six PSD estimates, modified covariance is found ideal for classifying the EEG sleep of a yoga practitioner with $97.3 \%$ accuracy. The extracted features from this PSD method shown 98\%, 97\%, and 98\% sensitivity, specificity and precision of the classifier, respectively Choosing and integrating features that show opposite actions works with KNN. The attributes included in this work show optimal and acceptable results with KNN. The quantitative results obtained through this proposed study showed distinguishable difference between the sleep patterns of yogic and non-yogic group. The study can be extended for developing computer aided tools for yoga assessment. Further the results contribute significantly to the growing field of yoga by improving sleep latency and quality, which in turn brings peace to mind and body.

\section{ACKNOWLEDGMENT}

We are also immensely grateful to S-VYASA Yoga University for providing us with the unpublished data. 


\section{REFERENCES}

Aboalayon, K., Faezipour, M., Almuhammadi, W., \& Moslehpour, S. (2016). Sleep Stage Classification Using EEG Signal Analysis: A Comprehensive Survey and New Investigation. Entropy (Basel, Switzerland), 18(9), 272. doi:10.3390/e18090272

Amin, H. U., Mumtaz, W. R., Subhani, A. N. M., Saad, M. S., \& Malik, A. (2017). Classification of EEG Signals Based on Pattern Recognition Approach. Frontiers in Computational Neuroscience, 11(103), 103. doi:10.3389/ fncom.2017.00103 PMID:29209190

Aunde, S., \& Shriram, R. (2014). PSD based Coherence Analysis of EEG Signals for Stroop Task. International Journal of Computer Applications, 95(16), 1-5.

Bankar, M., Chaudhari, S., \& Chaudhari, K. (2013). Impact of long term Yoga practice on sleep quality and quality of life in the elderly. Journal of Ayurveda and Integrative Medicine, 4(1), 28. doi:10.4103/0975-9476.109548 PMID:23741159

Dressler, O., Schneider, G., Stockmanns, G., \& Kochs, E. (2004). Awareness and the EEG power spectrum: Analysis of frequencies. British Journal of Anaesthesia, 93(6), 806-809. doi:10.1093/bja/aeh270 PMID:15377585

How Yoga Can Improve Your Sleep Quality and Help You Feel More Rested. (2020, July 28). Retrieved from https://www.sleepfoundation.org/articles/connection-between-yoga-and-better-sleep

Lee, M., \& Clarke, D. (n.d.). A Frequency Estimation Comparison between the Modified Covariance Method and the Fourier Transform. WSEAS Conferences.

Leonard, J. (2017, September 1). REM sleep: Definition, functions, the effects of alcohol, and disorder. Retrieved from https://www.medicalnewstoday.com/articles/247927

Lomb-Scargle Periodogram. (n.d.a). Retrieved June 7, 2020, from https://in.mathworks.com/help/signal/ref/ plomb.html

Lomb-Scargle Periodograms. (n.d.b). Retrieved June 7, 2020, from https://docs.astropy.org/en/stable/timeseries/ lombscargle.html

Maity, K., Rajesh, S., \& Venkatasamy, V. S. (2018). Efficacy of Sleep Special Technique on Young Healthy Yoga Practitioners. Indian Journal of Sleep Medicine, 13(3), 42-47. doi:10.5005/jp-journals-10069-0024

Multi-Taper Method (MTM). (n.d.). Retrieved June 7, 2020, from https://dept.atmos.ucla.edu/tcd/multi-tapermethod-mtm

Multitaper power spectral density estimate. (n.d.). Retrieved June 7, 2020, from https://in.mathworks.com/help/ signal/ref/pmtm.html

Mustafa, M. N., Taib, M. H., \& Murat, Z., \& Sulaiman, N. (2012). Comparison between KNN and ANN Classification in Brain Balancing Application via Spectrogram Image. Journal of Computer Science \& Computational Mathematics, 2(4), 17-22. doi:10.20967/jescm.2012.04.004

National Institute of Neurological Disorders and Stroke. (2019, August 13). Brain Basics: Understanding Sleep. Retrieved from https://www.ninds.nih.gov/Disorders/Patient-Caregiver-Education/Understanding-Sleep

Pereira, F., Mitchell, T., \& Botvinick, M. (2009). Machine learning classifiers and fMRI: A tutorial overview. NeuroImage, 45(1), S199-S209. doi:10.1016/j.neuroimage.2008.11.007 PMID:19070668

Rajak, B., Gupta, M., Bhatia, D., \& Mukhgerjee, A. (2017). Power Spectrum Density Analysis of EEG Signals in Spastic Cerebral Palsy Patients by Inducing r-TMS Therapy. International Journal of Biomedical Engineering and Technology, 4(1), 7-11.

Rajak, B. L., Gupta, M., Bhatia, D., Mukherjee, A., Paul, S., \& Sinha, T. K. (2016). Power Spectral Analysis of EEG as a Potential Marker in the Diagnosis of Spastic Cerebralpalsycases. International Journal of Biomedical Engineering and Science, 3(3), 23-29. doi:10.5121/ijbes.2016.3303

Ross, A., Friedmann, E., Bevans, M., \& Thomas, S. (2013). National survey of yoga practitioners: Mental and physical health benefits. Complementary Therapies in Medicine, 21(4), 313-323. doi:10.1016/j.ctim.2013.04.001 PMID:23876562 
Sabraj, M. (2013). Spectrum estimation of signals and analysis of sample-rate converter. Academic Press.

Verma, R., \& Dekar, R. (2018). Sleep Disorder Detection by Welch Algorithm Based PSD Analysis on EEG Signals. International Research Journal of Engineering Technology, 05(06), 858-865.

Wang, L.-L., Zheng, W.-L., Ma, H.-W., \& Lu, B.-L. (2016). Measuring sleep quality from EEG with machine learning approaches. International Joint Conference on Neural Networks (IJCNN). doi:10.1109/ IJCNN.2016.7727295

Welch, P. (1967). The use of fast Fourier transform for the estimation of power spectra: A method based on time averaging over short, modified periodograms. IEEE Transactions on Audio and Electroacoustics, 15(2), 70-73. doi:10.1109/TAU.1967.1161901

Welch's method. (2020, March 2). Retrieved June 7, 2020, from https://en.wikipedia.org/wiki/Welch's_method

Welch's power spectral density estimate. (n.d.). Retrieved June 7, 2020, from https://in.mathworks.com/help/ signal/ref/pwelch.html

Why so many methods of computing PSD? (n.d.). Retrieved June 7, 2020, from https://dsp.stackexchange.com/ questions/2096/why-so-many-methods-of-computing-psd

Basavaraj Hiremath did his undergraduate study in Medical Electronics Engineering at Ramaiah Institute of Technology and graduated in 2020. Basavaraj holds a keen interest in the field of biomedical signal processing and machine learning.

Meghana N has completed her undergraduate study in Medical Electronics Engineering from M.S Ramaiah Institute of Technology during 2020.

N. Sriraam received M.Tech degree(with distinction)in Biomedical Engineering from Manipal Institute of Technology(MIT),Manipal, India, in 2000, and the Ph.D. degree in Information Technology from the Multimedia University, Cyberjaya, Malaysia, in the area of biomedical signal processing, in 2007. He is currently working as Professor Department of Medical Electronics Engineering and Head,R\&D,, Ramaiah Institute of Technology, Bangalore, Inida. He is the author or coauthor of 150 articles published in journals and has been involved in several sponsored research projects. His current research interests include biomedical signal processing, data mining, neural networks. He is the editor-in-chief of International Journal of Biomedical and Clinical Engineering (IJBCE), USA and also reviewing panel member for Journals such as IEEE Transactions, IEEE Electronics letters, Elsevier publications. Dr. Sriraam is Senior Member of IEEE and IEEE Engineering in Medicine and Biology Society (EMBS), life member of telemedicine Society of India and member of medical computer society of India. Currently he is serving as Chairman of IEEE EMBS Chapter, Bangalore Section India.

Purnima B. R. received her Bachelor's degree in Medical Electronics from Dr. Ambedkar Institute of Technology, Bangalore, India in 2006 and completed her M.Sc. Engineering by Research under VTU in 2015. She is involved in the sleep study for the drowsiness detection in drivers through EEG analysis and in identification of significance of YOGA through the analysis of physiological signals. Her main areas of Interest are biomedical Signal Processing and statistics.

Nithin N. S. did his undergraduate study in Medical Electronics Engineering at Ramaiah Institute of Technology and graduated in 2020.. Nithin has a strong interest in biomedical instrumentation and signal processing.

Dr. V. Sureshbabu Venkatasamy is Assistant Professor at Swami Vivekananda Yoga Anusandhana Samsthana (S-VYASA Yoga University). He pursued his B.N.Y.S from Tamil Nadu, Dr. MGR Medical University and M.D in Yoga therapy \& Rehabilitation medicine from Swami Vivekananda Yoga Anusandhana Samsthana (S-VYASA Yoga University), Jigani. He has specialization in Yoga \& Sleep Medicine with enormous research interest in Sleep Medicine, Yoga-Therapy, Research Methods, Machine Learning Applications. 